\title{
L'Église face aux patriotes en 1837-1838
}

\section{Jean-Pierre Langlois}

Volume 51, 1984

URI : https://id.erudit.org/iderudit/1007449ar

DOI : https://doi.org/10.7202/1007449ar

Aller au sommaire du numéro

Éditeur(s)

Les Éditions Historia Ecclesiæ Catholicæ Canadensis Inc.

ISSN

0318-6172 (imprimé)

1927-7067 (numérique)

Découvrir la revue

Citer cet article

Langlois, J.P. (1984). L'Église face aux patriotes en 1837-1838. Sessions d'étude Société canadienne d'histoire de l'Église catholique, 51, 19-37.

https://doi.org/10.7202/1007449ar

\section{Résumé de l'article}

L'Église s'est montrée remarquablement active lors des troubles de 1837-1838. Cet article veut en rendre compte au mieux de la recherche historiographique actuelle. Mais il est bon de prendre conscience du substrat socio-religieux qui a cours à cette époque avant de tenter de situer la place de l'Église dans les composantes du processus révolutionnaire. Si l'Église a su tirer son épingle du jeu, c'est peut-être qu'elle n'avait plus rien à perdre...
Tous droits réservés @ Les Éditions Historia Ecclesiæ Catholicæ Canadensis Inc., 1984
Ce document est protégé par la loi sur le droit d'auteur. L'utilisation des services d'Érudit (y compris la reproduction) est assujettie à sa politique d'utilisation que vous pouvez consulter en ligne.

https://apropos.erudit.org/fr/usagers/politique-dutilisation/ 


\title{
L'Église face aux patriotes en 1837-1838
}

\author{
Jean-Pierre LANGLOIS \\ Hochelaga-Maisonneuve \\ RÉSUMÉ
}

L'Église s'est montrée remarquablement active lors des troubles de 1837-1838. Cet article veut en rendre compte au mieux de la recherche historiographique actuelle. Mais il est bon de prendre conscience du substrat socio-religieux qui a cours à cette époque avant de tenter de situer la place de l'Église dans les composantes du processus révolutionnaire. Si l'Église a su tirer son épingle du jeu, c'est peut-être qu' elle n'av'ait plus rien à perdre...

Le professeur Louis Rousseau a émis l'hypothèse que les efforts de la récente visite de Jean-Paul II au pays pourraient être comparés à l'impact qu'avait entraîné la prédication pastorale de $\mathrm{M}^{\mathrm{er}}$ de Forbin-Janson au Québec, au siècle dernier. À l'époque, il s'était agi d'une reprise considérable de l'influence de l'Église sur la réalité québécoise. D'aucuns affirment même qu'on assista dès lors à l'avènement d'une véritable emprise cléricale.

Au moment de la rébellion, l'idéologie libérale et les idéaux démocratiques des révolutions américaine et française avaient depuis longtemps modifié la culture ambiante, en exacerbant le nationalisme des «Canadiens». La crise économique provoquait de nécessaires transformations des structures de production agricole et commerçante. Elle suscitait de graves mécontentements chez la population, d'abord dans les campagnes, mais aussi dans les villes où allait débuter sous peu l'industrialisation. Des menaces démographiques vinrent accentuer l'insécurité de la majorité. Il fallut se résoudre au départ de milliers de Canadiens vers les États-Unis prospères, pendant que s'installaient dans les deux Canadas des centaines de milliers de Britanniques. 
Dans un univers travaillé par des courants de pensée autosuffisants, l'affrontement ne pouvait être évité. L'ultra-montanisme conservateur s'opposait ouvertement au libéralisme radical et démocratique.

J'ai l'intention d'aborder pour ma part un seul élément de compréhension tout en le situant dans un ensemble plus vaste ici esquissé: quelles furent les principales réactions de l'Église de la rive sud de Montréal au moment des troubles de 1837-1838? Cette Église n'est pas exclusivement celle du clergé. Pourtant, l'histoire de ses tıdèles du temps passé reste à faire. Aussi, ce n'est qu'incidemment qu'on saisira les comportements différents et les idéologies contradictoires qui y furent à l'oeuvre.

\section{LA SITUATION DE L'ÉGLISE À L'APPROCHE DE LA RÉBELLION}

On projette souvent sur l'Église des troubles de 1837-1838 une image de force et de prospérité qu'elle ne connaitra qu'après 1860 . C'est plutôt le contraire qui serait exact. Regardons-y de plus près.

L'encadrement religieux catholique a subi le creux de la vague au début du XIX ${ }^{c}$ siècle. De 33 ordinations entre 1775 et 1780 , on était tombé à 19 autour de 1800 , ce qui équivaut à une baisse de $43 \%{ }^{1}$. Si la tendance s'améliore graduellement par après, elle ne rattrapera la courbe de la population vers 1850 que grâce à l'arrivée de plusieurs communautés religieuses françaises au temps de $\mathrm{M}^{\mathrm{gr}}$ Bourget. C'est durant la décennie 1830-1840 que le phénomène atteint son déclin maximum: non pas un prêtre pour 1000 , mais un pour 1800 fidèles. «L'encadrement religieux n'a jamais été aussi faible au Québec ni avant, ni après cette période»².

D'où vient ce clergé raréfié? Dorénavant, il ne vient plus en majorité des villes, comme tout de suite après la conquête. Par exemple, Montréal ne donnera pas un prêtre par année à son diocèse entre 1800 et 1840 . C'est la campagne environnante qui prend la relève: 22 prêtres pour la ville, 78 pour le plat pays d'alentour. «Comme la répartition de la population entre la ville et la campagne est demeurée à peu près constante tout au long de la période pour l'ensemble du Québec, il faut conclure à la mise en place progressive de la zone rurale comme lieu privilégié de recrutement $»^{3}$.

\footnotetext{
' Serge GAGNON et Louise LEBEL, «Le milieu d'origine du clergé canadien. 1775-1840: mythes et réalités». Rev'ue d' histoire de l'Amérique française, (RHAF),37, n" 3 (décembre 1983), p. 376, tableau 1.

=Ibid., p. 377 .

'Ibid., p. 380, tableau 4.
} 
Il faut dire que la ville de Montréal comptait à l'époque, une majorité anglophone, en grande partie protestante. De plus, c'est en ville que se répandent les idées philosophiques à la mode, le libéralisme, le nationalisme patriotique, le rationalisme voltairien: «il existe dans les familles bourgeoises un préjugé défavorable à l'endroit de la prêtrise» ${ }^{4}$.

Après 1800 , les chefs de l'Église ne purent donc compter sur l'élite cultivée (professionnels, seigneurs, fonctionnaires, commerçants) pour défendre son statut dans la société. Le clergé se recruta plutôt dans les classes moyennes, du côté des fils d'artisans et de cultivateurs. Ridiculisé ou ignoré par l'élite savante et la petite bourgeoisie francophone, ce clergé paysan se montra davantage préoccupé par les réactions du peuple d'où il était issu, que par les appels enflammés des députés libéraux révoltés en 1837-1838. C'est encore lui qui donnera plus tard l'impulsion à la colonisation de l'arrière-pays.

D'après les historiens, le peuple canadien au début du XIX ${ }^{e}$ siècle est généralement inculte et superstitieux. Ces gens sont frondeurs et près de leurs sous. Les saisons de récolte sont courtes: il ne faut pas les perdre en fêtes d'obligation chômées. L'hiver est dur: il faut canaliser l'inactivité dans des charivaris qui passent le temps. L'autorité publique est éloignée des campagnes, les distances à parcourir sont énormes pour l'époque, la population paysanne est endettée et généralement mécontente de se faire exploiter par des intermédiaires, de langue étrangère par surcroit.

Guider un troupeau aussi récalcitrant avec une poignée de prêtres n'était sans doute pas une sinécure. On rapporte que des fabriques devaient même engager des fiers-à-bras pour maintenir l'ordre dans l'église! «Les évêques tentent de persuader patiemment les habitants rebelles et déplacent les curés brouillons qui cherchent querelle à leurs marguilliers — bien que la situation inverse semble la plus fréquente»s.

Dans les chansons politiques de cette époque troublée, il est souvent question de la nation «canadienne». C'est une race de gens têtus, c'est un peuple indéracinable, qui cultive précieusement le souvenir de sa mèrepatrie française, et qui salue tous ses événements révolutionnaires comme autant de germes de sa libération prochaine. On est toujours à préparer la prochaine revanche... du moins en chanson'

\footnotetext{
+ Ibid., p. 392.

5 Jean-Pierre WALLOT, "L'Église canadienne et les laïcs au début du XIX' siècle», Pierre HURTUBISE et al., Le lä̈c dans l'Église canadienne-française de 1830 à nos jours, Montréal, Fides, 1972, p. 91.

'Maurice CARRIER et Monique VACHON, Chansons politiques du Québec, 1834-1858, Montréal, Leméac, 1979, tome 2, 450 p.
} 
Jean-Pierre Wallot a bien résumé la situation de faiblesse - de survie même - qui est celle de l'Église catholique à l'approche des troubles de 1837-1838:

Au début du XIX' siècle, il n'y a pas d'Église triomphante, forte et prospère, pas de clergé très influent et dominateur, pas de grappes de fidèles particulièrement religieux et vertueux. (. . .) L'Église déploie des efforts inouïs simplement pour subsister au milieu de l'hostilité ou de l'indifférence de l'élite instruite et de l'indépendance du peuple.

À mon avis, on ne s'attacherait pas autant à l'attitude de l'Église pendant la rébellion de 1837-1838, si elle n'était pas devenue par la suite le point de référence obligé de la société québécoise. On ne sera plus surpris d'imaginer dorénavant que la condamnation de la souveraineté du peuple et de la désobéissance civile par l'Église hiérarchique avant les troubles de 1837 n'ait que peu influencé le cours des événements. La pente n'était pas encore remontée, mais le vacuum politique laissé par l'échec du parti patriote y contribuera puissamment.

\section{TENSIONS EXTRÊMES AU BAS-CANADA EN 1837}

Essayons maintenant de nous faire une impression juste de l'ambiance générale qui entoura la rébellion au Bas-Canada. Les troubles de 1837-1838 se situent dans l'éveil généralisé des nationalismes issus du siècle des lumières et des guerres napoléoniennes. La France s'est affranchie de la monarchie absolue, les États-Unis se sont séparés de l'Angleterre, les États sud-américains de l'Espagne, la Belgique de la Hollande. Un jour, ce sera le tour de l'Italie et de l'Allemagne. Le peuple canadien n'était pas insensible à ce vaste mouvement de libérations nationales.

C'est une nouvelle petite bourgeoisie francophone, composée de notaires, d'avocats, de médecins et de commerçants qui s'est imprégnée de l'idéologie nationaliste, républicaine et libérale. Opposée à la haute bourgeoisie capitaliste britannique qui contrôle avec les militaires le pouvoir exécutif, elle a trouvé, depuis 1791 , dans la représentation parlementaire le moyen de véhiculer sa pensée, ses objectifs, ses intérêts. Tout cela se concrétisera dans la formation bien structurée du parti patriote, qui prendra la relève du parti canadien en 1826 .

L'ensemble de la population canadienne est sensible à cette approche autonomiste, de plus en plus radicale. Le vieux fonds normand n'avait

Jean-Pierre WALLOT. «l'Église canadienne...». p. 91. 
jamais tout à fait acquiescé à la conquête, à tout le moins en paroles et en chansons. Mais justement cet attachement aux valeurs traditionnelles, y compris à la religion qui faisait partie de l'identité nationale, venait par ailleurs freiner inconsciemment l'appel aux changements lancé par les patriotes. Il fallait cimenter l'opposition des masses paysannes et de la bourgeoisie locale, en lui présentant un adversaire commun: le Riche, l'Étranger, l'Anglais.

Les députés de la Chambre d'Assemblée se montrèrent de plus en plus réticents à voter le budget du gouvernement, sans le contrôler de quelque façon. Ils entendaient surveiller non seulement les dépenses affectées à l'aménagement du territoire ou au bien-être de la population (par exemple, la voirie) - ce qu'on appelait les subsides, mais aussi la liste civile, c'est-à-dire l'administration et ses deux Conseils (exécutif et législatif) qui appuyés par Londres, se refusèrent à céder aux revendications des parlementaires.

Peu à peu, les modérés du parti patriote se verront réduits au silence, voire accusés de trahison. La cause patriote ne permettait plus à personne de rester indifférent. Des causes sous-jacentes étayaient cette tension considérable. Londres cherchait à unir les deux Canadas afin de mettre en minorité les Canadiens face aux immigrants britanniques, en plus de partager l'énorme dette du Haut-Canada en deux. Les marchands anglais monopolisaient le commerce extérieur et souhaitaient des investissements majeurs en faveur de la navigation fluviale, alors même que le commerce intérieur échappait peu à peu aux commerçants canadiens, au profit de la Compagnie de la Baie d'Hudson. Une crise économique entre 1833 et 1836 rendit la situation encore plus pénible.

À cela, s'ajoute une crise agricole. Les terres sont limitées et de peu d'étendue. Les cultures du blé s'étiolent à cause de la stagnation de techniques d'agriculture et de l'épuisement consécutif des sols. Les nouvelles terres sont vendues à des prix trop élevés pour la plupart des Canadiens. Les cultivateurs voient leurs fardeaux s'alourdir pour permettre aux seigneurs ruraux de survivre.

La pression démographique se résorbe dans l'émigration vers les ÉtatsUnis, mais cela disloquait le clan familial jusqu'alors préservé. Entre 1830 et $1844,40,000$ Canadiens s'expatrièrent outre-frontières. Pendant ce temps, de 1820 à $1850,850,000$ Britanniques immigraient au pays.

En 1834, le parti patriote présenta en Chambre 92 Résolutions. Essentiellement, il exigeait, au nom du peuple, une représentation majoritaire des 
Canadiens aux Conseils exécutif et législatif, l'électivité du Conseil législatif et la responsabilité du Conseil exécutif devant la Chambre d'Assemblée plutôt que devant la métropole coloniale. On s'opposa à ces demandes dans les milieux conservateurs, au Bas-Canada et à Londres. En 1837, devant l'impossibilité de faire des compromis acceptables de part et d'autre, Londres autorisa le gouverneur du Bas-Canada à utiliser tous les fonds publics sans l'accord des députés trop intransigeants.

Dès $1 \overline{8} 34$, i'évéque de Mĩontréai jugeait ia situation préoccupante. î écrivait à son cousin. Denis-Benjamin Viger:

Déjà nos Assemblées de comtés, pour adhérer à la dernière requête de la Chambre d'Assemblée sur les nouveaux griefs de la province, deviennent passablement tumultueuses à raison de la chaleur des partis; et je vois qu'on commence, même en campagne, à y introduire des boulés pour vaincre par l'argument du poing. ${ }^{\text {}}$

Cette effervescence débridée culmina dans les assemblées de protestation de l'annéc 1837. À St-Ours le 7 mai, à St-Charles le 23 octobre, ailleurs entre temps, les chefs patriotes défièrent ouvertement les autorités politiques en place. On engageait les citoyens à boycotter les produits anglais, et même à s'adonner à la contrebande, afin de tarir les sources de revenus de l'État. À la fin, certains prônèrent la révolte armée pour venir à bout du gouvernement.

On montait en épingle la souveraineté populaire. Dans son livre $\mathrm{Pa}$ roles d'un crovant, le célèbre Lamennais en avait fait une description lyrique quasi incantatoire. Elle était la garante de la liberté des peuples opprimés et la crainte suprême des tyrannies. La voix populaire n'était nulle autre que la voix de Dieu. Pour Lamennais, «le consentement de l'humanité est l'unique fondement du vrai et la souveraineté du peuple est l'unique source de l'autorité ${ }^{4}$. Son pamphlet fut édité au pays en 1836. Le journal patriote La Minerve osa écrire le 7 août 1837:

La souveraineté du peuple est un principe incontestable et incontesté. Il ne s'agit plus que d'en tirer les conséquences; et parmi ces conséquences, se trouve tout naturellement celle de modifier ou de changer par le peuple la forme de son gouvernement."

\footnotetext{
${ }^{8}$ Lartigue à D.-B. Viger. 15 avril 1834. cité dans Gilles CHAUSSÉ. Jéan-Jacqués Lartigue'. premier évêque de Montréal. Montréal. Fides. 1980, p. 192.

"Germain LESAGE. "Un fil d'Ariane: la pensée pastorale des évêques canadiens-français". Pierre HURTUBISE et al.. Le Laï'. p. 20.

"La Minerve. 7 août 1837. cité dans G. CHAUSSÉ. Jean-Jacques Lartigue'. p. 202.
} 
Lors de la session de la Chambre d'Assemblée de 1837 à Québec, le gouverneur Gosford fit face à des députés véhéments, vêtus à la canadienne, et qui se crurent déliés de leur serment de loyauté et d'obéissance à la Couronne lorsqu'ils virent qu'on allait passer outre à leur vote négatif quant à l'attribution des fonds publics. C'en était fini de la Constitution de 1791 . Mais le gouverneur temporisa encore longtemps avant de se rendre à l'évidence et de faire arrêter les fauteurs de troubles:

On trouve généralement ici que le Gouvernement laisse bien marcher les choses comme elles veulent aller; et l'on croit remarquer beaucoup d'apathie parmi ses officiers, surtout ceux de robe."

À St-Charles-sur-Richelieu s'était tenue une dernière assemblée de protestation, la plus virulente, une des plus nombreuses. Le curé Magloire Blanchet - qui sera arrêté parce que suspect, après le soulèvement — prit la peine d'écrire ses impressions aux autorités politiques. Le ton anxieux de sa lettre au gouverneur, rédigée le 9 novembre 1837 - deux semaines avant la bataille - laisse entrevoir que le point de non-retour est tout proche.

Je crois connaître assez l'opinion de la population circonvoisine pour vous dire que le danger est imminent, qu'il n'y a pas de temps à perdre, si vous avez quelque chose à faire pour le bonheur de Canadiens, (...) l'excitation est à son comble. Il n'y a pour ainsi dire qu'une voix pour condamner la conduite du Gouvernement (...) Le mouvement est tel que, pour ma part, je doute beaucoup que ceux qui l'ont excité puissent l'arrêter, quand ils en auraient la volonté.'"

Les dispositions des participants chauffés à blanc étaient «celles d'hommes persuadés qu'il fallait faire les plus grands efforts» pour réparer les injustices et mettre un terme aux souffrances du pays ${ }^{13}$. L'Église ne pouvait rester indifférente. L'élite appelait à la révolte, le peuple se regroupait pour la suivre. Il fallait réagir.

\section{LES INTERVENTIONS DE L'ÉGLISE DURANT LES TROUBLES DE 1837-1838}

L'évêque de Montréal de l'époque, $\mathrm{M}^{\mathrm{gr}} \mathrm{Jean}-J a c q u e s ~ L a r t i g u e$, n'est pas un homme facile. Sa vertu principale n'est pas la souplesse. Il ne ménage

\footnotetext{
"Lartigue à Signay, évêque de Québec, 2 novembre 1837, Archives de la Chancellerie de l'Archidiocèse de Montréal (ACAM), Registre des lettres de $M^{\text {er }}$ Lartigue (RLL), t. 8. p. 445.

12 Blanchet à Gosford, gouverneur. St-Charles-sur-Richelieu, 9 novembre 1837. ACAM. 295.010, 838-17B.

${ }^{1.3}$ Blanchet à Bourget, coadjuteur de Montréal, 13 janvier 1838, ACAM, 295.101, 838-15B.
} 
ni ses prêtres ni les fidèles de son diocèse. Cousin de Papineau et des Viger, il est foncièrement nationaliste. Même son nationalisme est conservateur. Mais il permettra à l’Église québécoise de prendre son véritable envol en la libérant définitivement de la mainmise étatique coloniale et il saura profiter des erreurs des leaders libéraux pour asseoir son autorité et son influence décisive auprès du peuple canadien. Doué d'un dynamisme débordant, il était sans doute la figure de proue de l'Église du Bas-Canada. Homme flamboyant, il commandait le respect, sinon l'obéissance.

Dans la conception de $\mathrm{M}^{\mathrm{r}}$ Lartigue, le laïc devait être ur chrétien entièrement soumis aux directives de son évêque dans l'ordre moral et dans les divers domaines temporels où l'Église se croyait concernée, tels l'éducation, les paroisses et les fabriques. L'Église, selon $M *$ Lartigue, avait le devoir de former les laïcs pour qu'ils devinssent des exécutants fidèles des volontés et des décisions de la hiérarchie. Ainsi, il seraient en mesure, sous la direction de l'épiscopat et du clergé, de lutter plus efficacement contre les ennemis de l'Église et contre la diffusion des idées fausses et pernicieuses $^{1+}$.

Il s'agit là en réalité de l'idéologie ultramontaine déjà en oeuvre. En temps de crise, elle renforce l'autorité en l'identifiant à la volonté divine. On consacra son apothéose avec la définition dogmatique de l'infaillibilité pontificale en 1870 .

$\mathrm{M}^{\mathrm{r}}$ Lartigue fut l'un des premiers adeptes de l'ultramontanisme. En conséquence, il exigera la soumission et l'obéissance autant de son clergé que de ses fidèles, spécialement durant les troubles de 1837-1838. Mais cette attitude n'était pas nouvelle chez lui. En 1829, il était opposé à ce que des laïcs de son diocèse fassent pression à Rome en sa faveur; «Je n'entendrai jamais à inviter les laïcs, écrivait-il à $\mathrm{M}^{\mathrm{gr}}$ Panet de $Q u e ́ b e c$, de se mêler de l'érection d'un évêché à Montréal parce que ce n'est pas de leur compétence, que c'est contre les principes, et que ce serait donner gain de cause au curé Chaboillez qui, dans ses pamphlets, a soutenu la nécessité de leur concours» ${ }^{15}$.

Si l'Église dans son ensemble n'avait pas repris du poil de la bête, $\mathrm{M}^{\mathrm{gr}}$ de Montréal ne se laissait pas impressionner outre mesure.

\footnotetext{
${ }^{1+}$ G. CHAUSSÉ, «L'attitude de l'épiscopat envers le laïcat vers 1830». Pierre HURTUBISE et al., Le Laïc, p. 107.

${ }^{15}$ Lartigue à Panet, évêque de Québec; 17 février 1829, ACAM. RLL, 5: 27.
} 


\section{Premières interventions prudentes de l'évêque de Montréal}

Les premières interventions de $\mathrm{M}^{\text {qr }}$ Lartigue, se révélèrent très prudentes. On sent que ses prises de position vinrent lorsqu'il sentait qu'il ne pouvait plus se taire. Durant l'été 1837 , pendant que se développait le mouvement de contestation politique, l'évêque indique privément à différents membres du clergé, dans ses lettres personnelles, la conduite qu'il leur enjoignait de suivre avec les patriotes. En gros, on peut dire que $\mathbf{M}^{\mathrm{q}} \mathrm{Larti}-$ gue incitait ses prêtres à demeurer prudents en chaire et à refuser de prendre parti dans les discussions politiques.

Il ne faut point parler en chaire d'affaires politiques ni par conséquent d'assemblées, mais bien contre ceux qui perdent la messe d'obligation (du dimanche) pour de pareilles causes. ${ }^{16}$

$\mathrm{M}^{\mathrm{rr}}$ Lartigue profita aussi d'une circonstance très favorable pour proposer à tout le clergé du diocèse de Montréal des consignes précises face aux exagérations des patriotes. Le 25 juillet 1837, lors d'un banquet qui suivit le sacre de son coadjuteur, $\mathrm{M}^{\mathrm{gr}}$ Bourget, il prit la parole pour faire savoir:

1- que les pasteurs devraient faire tous leurs efforts pour rétablir la charité et l'union parmi leurs ouailles;

2- qu'il n'est jamais permis de se révolter contre l'autorité légitime, ni de transgresser les lois du pays (cf. la contrebande avec les États-Unis);

3- que les prêtres ne devraient pas absoudre ceux qui enseignaient, ou pire mettaient en oeuvre de tels principes. ${ }^{17}$

Les événements se précipitèrent. On en vint bientôt à prôner l'emploi de la force pour vaincre les résistances du gouvernement colonial anglais. Comme le climat d'agitation prenait de plus en plus d'ampleur, $\mathrm{M}^{\mathrm{kr}}$ Lartigue se crut obligé, de par sa fonction d'évêque, de rappeler publiquement à tous ses fidèles les devoirs inhérents au dépôt révélé, selon lui. Dans un mandement daté du 24 octobre 1837, à peine un mois avant le début des troubles proprement dit, l'évêque rappelait que d'après l'Écriture $(\mathrm{Rm} 13$ et $l \mathrm{Pi}$ 2) et certains textes pontificaux de l'époque (Grégoire XVI), l'autorité civile établie tenait sa puissance de Dieu lui-même. Celui qui s'opposait à la bonne marche de l'État s'attirait par le fait même la condamnation divine. Il ne pouvait plus s'agir désormais pour tout bon catholique que d'obéir aux lois de la religion ou d'abandonner l'Église.

\footnotetext{
${ }^{16}$ Lartigue à Quévillon, St-Polycarpe de Valleyfield, 6 septembre 1837, ACAM, RLL, 8: 419.

${ }^{17} L$ 'Ami du Peuple, 26 juillet 1837, cité dans Gérard FILTEAU, Histoire des Patriotes, 3 vol. Montréal, A.C.F., 1938, t. 2, p. 132.
} 
La lecture du mandement épiscopal en chaire le dimanche suivant provoqua certains remous, ici et là. Les journaux patriotes grossirent encore les oppositions. $\mathrm{M}^{\mathrm{gr}}$ Bourget, alors en visite à Chambly, avait été à même de mesurer l'impopularité des directives de son évêque. Au moment de la lecture du mandement, plusieurs personnes étaient sorties de l'église en guise de protestation; puis une vingtaine de mécontents manifestèrent sur la place en criant «À bas le mandement ${ }^{1 \times}$. À St-Charles, au moment où le curé Blanchet commença la lecture du mandement, plus de la moitié des fidèles quittaient l'église pour ne revenir qu'une fois le mandemeni iu ${ }^{10}$.

Pour se justifier, $\mathrm{M}^{\mathrm{gr}}$ Lartigue publia incognito, quelques semaines plus tard, une Défense du Mandement. Il y répétait qu'on ne pouvait plus se considérer catholique et soutenir des principes révolutionnaires. Il accusait en outre les chefs patriotes de flatter le peuple pour mieux le jeter dans la guerre civile. Affirmant au tout début de son texte ses liens de sang avec la population et son indépendance proverbiale face au gouvernement anglais, l'évêque lançait son appel à la soumission à l'autorité établie, avant tout par devoir de conscience. C'est ce qu'il écrivait un peu plus tard:

$\mathrm{J}$ 'ignore encore si mon dernier mandement fera quelque impression sur nos gens: tout ce que je sais est que j'ai fait mon devoir quand je l'ai cru opportun; et je resterai ensuite tranquille..$^{\text {"n }}$

\section{Des prêtres proches de leur peuple}

En règle générale, les prêtres de la région refusèrent de se laisser entraîner à prendre position du côté des patriotes dans les discussions, même si certains avaient des options nationalistes. M. Magloire Blanchet, le curé de St-Charles, expliquait à lord Gosford, gouverneur du Bas-Canada, pourquoi les prêtres restaient silencieux: «Il ne faut plus compter sur les messieurs du clergé, pour arrêter le mouvement populaire dans les environs (... vous savez que les pasteurs ne peuvent se séparer de leurs ouailles $»^{21}$.

Je pense pourtant à une intervention significative des curés de la région du Richelieu. On se rappelle que c'est d'abord là qu'allait éclater la rébellion de 1837-1838. Le supérieur du Collège de St-Hyacinthe, M. Jean-Charles Prince, prit l'initiative de les réunir, au début du mois de novembre 1837, pour contrer le mécontement qu'avait suscité la lecture du mandement de

\footnotetext{
${ }^{1 *}$ Lartigue à Demers, St-Denis-sur-Richelieu, 30 octobre 1837, ACAM, RLL. 8: 443.

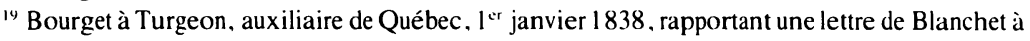

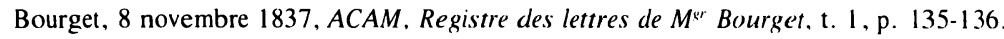

2" Lartigue à Signay, 2 novembre 1837. ACAM, RLL, 8: 444-445.

${ }^{21}$ Blanchet à Gosford, 9 novembre 1837, ACAM, 295.101, 838-17B.
} 
$\mathrm{M}^{\mathrm{sr}}$ Lartigue chez la population des environs. Ils proposèrent à l'évêque de Montréal une démarche spéciale auprès des autorités politiques et religieuses afin de les amener à se pencher avec plus de célérité sur les problèmes qui agitaient le Bas-Canada. Ainsi on contrebalançait l'apparente approbation de la conduite du gouvernement, en présidant une requête faisant connaître, sur un ton franchement nationaliste, les besoins réels des Canadiens et leur désir d'être traités avec justice et bienveillance.

M"r Lartigue accueillit favorablement ce projet et s'en fit même le promoteur auprès du gouverneur Gosford et de l'évêque de Québec. Il fit paraître en plus une lettre circulaire dans le but «d'engager, selon ses propres mots, notre clergé à signer avec nous» la pétition"2. Mais avant que les signatures des prêtres du diocèse de Montréal ne fussent recueillies et que $\mathrm{M}^{\mathrm{gr}}$ Signay de Québec n'eût finalement donné son approbation à cette démarche, l'insurrection armée avait déjà eu lieu, rendant périmée par le fait même la requête du clergé de Montréal.

\section{Dans le feu de l'action}

La lutte armée atteignit son paroxisme entre le 17 novembre et le 15 décembre 1837. Deux régions furent particulièrement touchées par la rébellion. C'est aux villages de St-Denis et de St-Charles, dans la vallée du Richelieu, au sud de Montréal, que s'engagèrent d'abord les combats. Les hostilités se poursuivirent ensuite sur la rive nord, du côté de St-Eustache et de St-Benoit. Après une première victoire le 23 novembre à St-Denis, les patriotes, mal armés et souvent indisciplinés, abandonnés de surcroît par la plupart de leurs chefs, furent régulièrement battus par les troupes britanniques, leurs villages incendiés et 495 d'entre eux se retrouvèrent derrière les barreaux de la prison de Montréal. Ainsi environ 5,000 Canadiens, soit 2\% des catholiques du diocèse, furent impliqués dans la rébellion.

$\mathrm{M}^{\mathrm{gr}}$ Lartigue critiqua le curé de St-Denis, M. François-Xavier Demers, pour avoir fait preuve de faiblesse durant cette période critique. Il avait en effet livré le coffre de la Fabrique aux insurgés devant leurs menaces et s'était enfui par après de sa paroisse devant l'avance des troupes anglaises. L'évêque le pria de regagner le plus tôt possible son village, tout au moins dès que le danger serait disparu.

"Circulaire de Lartigue aux prêtres du diocèse de Montréal, 17 novembre 1837, ACAM, RLL. 8: 449 . 
Un autre curé eut sa part de déboires durant ces événements. Il s'agit du curé Magloire Blanchet, de St-Charles-sur-Richelieu. qui fut soupçonné d'intelligence avec les rebelles. Après le pillage de son presbytère, son église fut profanée et lui-même incarcéré pendant plus de trois mois. Même s'il avait ouvertement blâmé la révolte et refusé de prêter secours aux insurgés, il avait voulu rejoindre le champ de bataille pour soigner les blessés du camp patriote. $\mathrm{M}^{\mathrm{rr}}$ Lartigue prit fait et cause pour lui. se déclarant convaincu que $\mathrm{M}$. Blanchet ne pouvait être coupable du crime de haute trahison. bien qu ii ait pu avoir été queique peu imprudent. ii suggéra donc fortement de le relâcher.

Par ailleurs, on ne peut finalement laisser de côté l'influence des sulpiciens français de Montréal. Composé en grande partie de prêtres ayant connu les effets désastreux de la Révolution française et des guerres napoléoniennes, le groupe des sulpiciens, solidement implanté dans la ville de Montréal, amena beaucoup de curés et de nombreux laïcs à réagir contre le mouvement insurrectionnel. Bien que cette influence ait été diffuse, elle fut si grande au dire du général anglais Colborne que le Séminaire avait plus contribué, selon lui, à abattre la rébellion que tous ses régiments ${ }^{23}$.

\section{La condamnation des insurgés}

$M^{\mathrm{m}}$ Lartigue protesta plus d'une fois contre les pillages et les exactions des soldats ou des volontaires orangistes, auprès du gouverneur ou de ses représentants. Alors que $\mathrm{M}^{\mathrm{gr}}$ Bourget voyait la situation sous un angle pessimiste, l'évêque en titre se montre plus modéré: «ll y a maintenant ici, écrit-il à l'un de ses correspondants, une réaction terrible des prétendus loyaux (sujets de Sa Majesté) contre les Canadiens catholiques; et ces excès peuvent nuire beaucoup au Gouvernement; il ne faut jamais pousser personne au désespoir ${ }^{2+}$. Alors que les patriotes récoltaient maintenant les fruits amers de leur désobéissance civile, $M^{\text {ør }}$ Lartigue multiplia ses démarches en faveur de chacun. Il engagea ainsi $\mathrm{M}^{\mathrm{r}}$ Bourget à visiter les prisonniers politiques et à soulager la misère des familles éprouvées. Il encouragea encore les prêtres de son diocèse à faire signer à la population des adresses de loyauté à la reine, afin d'apaiser la colère des autorités civiles.

D'un autre côté, il refusa aux insurgés morts sur le champ de bataille, les armes à la main, le droit à la sépulture ecclésiastique. Car ces rebelles

\footnotetext{
${ }^{23}$ Quiblier. supérieur canadien des Sulpiciens, à Carrière, supérieur général français des Sulpiciens, $1^{\text {er }}$ août 1838, cité dans Gilles CHAUSSÉ. Jean-Jacques Lartigue. p. 218.

${ }^{2+}$ Lartigue à Turgeon. 8 janvier 1838. ACAM, RLL. 9: 17.
} 
décédés au cours des combats étaient réputés être des pécheurs publics morts sans repentir. Pourtant il incita tout de même ses curés à prier privément "pour le repos de leurs âmes». Les peines canoniques que l'évêque de Montréal décréta sont donc le résultat d'une analyse dogmatique de la situation des insurgés. Il résuma ses consignes ainsi:

[...] De n'admettre aux sacrements de l'Église, même à l'heure de la mort, sans une réparation préalable, aucun de ceux qui se sont montrés scandaleusement rebelles, et de refuser la sépulture ecclésiastique à ceux qui mourraient sans s'être acquittés de cette juste réparation."s

Réagissant à l'échec du mouvement révolutionnaire, $\mathrm{M}^{\mathrm{gr}}$ Lartigue rendit publique le 8 janvier 1838 son analyse des troubles lors de la parution d'un second mandement. Il y montrait comment les Canadiens auraient eu avantage à suivre sa ligne de pensée personnelle. Par contre, réapparaît clairement dans ce document la tendance loyaliste et contre-révolutionnaire de l'évêque de Montréal, favorable plus que jamais au respect de la loi et de l'ordre établi.

Somme toute, selon les évêques de Montréal, l'esprit religieux du peuple avart beaucoup souffert durant la rébellion. Les chefs patriotes avaient attaqué le clergé pour diminuer son influence auprès de la population. Berné par ses chefs, le peuple s'était laissé aller à des gestes de cruauté peu prévisibles. La misère gagna beaucoup de villages durement châtiés à cause de leur accueil aux rebelles. Pillages et incendies ruinèrent des comtés entiers.

Que nous reste-t-il de leurs promesses (celles des patriotes), sinon l'incendie de nos maisons et de nos églises, la mort de quelques-uns de nos amis et de nos proches, la plus extrême indigence pour un grand nombre d'entre nous? $?^{26}$

L'évêque de Montréal prévoyait les conséquences néfastes de la rébellion. Il craignait que les Anglais profitassent de l'occasion pour tenter de nouveau l'assimilation des Canadiens en les privant de leurs lois propres, de leurs droits acquis, de leur langue et de leur religion ${ }^{27}$.

\footnotetext{
25 Mandements, lettres pastorales, circulaires et autres documents publiés dans le diocèse de Montréal, (MEM) Montréal, Le Nouveau Monde, 1869, tome 1, p. 29, 8 janvier 1838.

26 Ibid., p. 25.

${ }^{27}$ Lartigue à Griffiths, évêque de Londres, 27 avril 1839, ACAM, RLL, 9: 175.
} 


\section{La lutte du clergé contre l'Union}

Lord Durham fut nommé non seulement pour pacifier le pays, mais aussi pour proposer des solutions à l'imbroglio politique des colonies britanniques d'Amérique du Nord. À la fin de juin 1838. il prit sur lui d'amnistier les prisonniers politiques à l'exception de huit d'entre eux qui furent exilés aux Bermudes. Mais il résigna rapidement son poste parce qu'on lui avait reproché, en haut lieu, le relâchement des patriotes emprisonnés.

Son départ sonna l'heure d'une seconde révolte. Les insurgés s'emparèrent d'un vaisseau gouvernemental à Beauharnois, au début de novembre 1838, et tentèrent de s'emparer de la réserve de Caughnawaga; puis, devant leur échec, se dirigèrent vers Napierville. Colborne, militaire devenu chef intérimaire du pays, marcha contre ces 2,000 rebelles, à la tête de 7,000 soldats et volontaires orangistes. Il les atteignit près de la frontière américaine et les dispersa aisément.

Il fit ensuite goûter sa répression à la population de la région située entre Laprairie, Chateauguay, St-Constant, Napierville et les frontières. Il y gagna le surnom de «Vieux Brûlot». On écroua 850 personnes, dont 108 subirent un procès. 99 furent condamnées à mort, dont 12 furent exécutées à la prison du Pied-du-Courant, en décembre 1838 et janvier 1839. Plusieurs autres furent exilées, cette fois en Australie.

$\mathrm{M}^{\mathrm{gr}}$ Lartigue dut s'aliter peu après son séjour à Québec au moment des troubles de novembre 1838 . C'est à $\mathrm{M}^{\mathrm{gr}}$ Bourget, son coadjuteur, de déployer dorénavant beaucoup d'énergie auprès des autorités civiles. Mais il n'est guère douteux que ces différentes démarches, en particulier celles en faveur des condamnés politiques, aient eu l'assentiment de son supérieur immédiat. $\mathrm{M}^{\mathrm{rr}}$ Bourget visita donc régulièrement les patriotes prisonniers. Il pria les curés des détenus de venir les rencontrer et de les réconforter. Après les premières condamnations à mort, il fit intervenir le clergé de Montréal en faveur de ces malheureux:

Le clergé exerçant un ministère de miséricorde, nous allons nous adresser à Son Excellence pour la supplier d'user d'indulgence, dans l'espérance que cet Acte de Clémence, tout en sauvant des malheureux conciliera l'affection publique envers un Gouvernement vraiment paternel..$^{28}$

Il se préoccupa entre autres de faire assainir le climat de la prison de Montréal, qui avait besoin d'urgentes réformes.

${ }_{28}$ Bourget à Mondelet, secrétaire du Conseil spécial, 17 décembre 1838, ACAM, RLB. 1: 253. 
Durant ce temps, Durham transmit son rapport d'enquête au Secrétaire londonien aux Colonies. Il y proposait quelque chose d'inédit pour le temps, c'est-à-dire l'instauration d'un Canada uni avec gouvernement responsable. Cela impliquait que le parti majoritaire à la nouvelle Chambre d'Assemblée choisirait les membres de l'exécutif de la colonie. Cet aspect du rapport sur l'organisation politique d'une colonie, fort nouveau en Europe, fut laissé dans l'ombre par les Canadiens, qui remarquèrent davantage dans le rapport la volonté de Durham d'assimiler les francophones. Après que celui-ci eût démontré que la race supérieure, celle des Britanniques, devait dominer, il recommanda la subordination politique des Canadiens français soit en confédérant les six colonies d'Amérique Britannique du Nord, soit en unissant seulement deux d'entre elles: le Haut-Canada et le Bas-Canada. De fait, il préférait cette dernière éventualité, quitte à ce que les autres colonies s'y joignent par la suite à leur gré.

La population canadienne-française ne réussit cependant pas à réagir en masse, ses chefs politiques étant en prison ou en exil. Certains d'entre eux qui étaient demeurés au pays, considéraient de fait l'Union comme une étape en vue d'accélérer la séparation coloniale du Canada d'avec la GrandeBretagne. $\mathrm{M}^{\mathrm{r}}$ Lartigue fut le premier à intervenir. Il écrivit à Londres à l'ancien gouverneur Gosford et à son représentant personnel auprès du gouvernement, le vicaire apostolique de Londres. À tous deux, il demandait instamment de faire en sorte que le Parlement n'endossât pas le rapport Durham. Il espérait que le Parlement ne priverait pas les Canadiens français de leur législation civile française propre, de leur langue, de leurs prérogatives en éducation et de leur religion catholique.

Le nouveau gouverneur général, arrivé au pays en octobre 1839, Thompson, fit endosser la mesure de l'Union par son Conseil spécial du Bas-Canada, composé seulement de Canadiens anglais, à l'insu de la population, puis par la Chambre d'Assemblée du Haut-Canada; il transmit à Londres en février 1840 un projet d'union. Entre temps, $\mathrm{M}^{\mathrm{gr}}$ Lartigue était de nouveau intervenu à Londres auprès de Gosford et $\mathrm{M}^{\mathrm{r}} \mathrm{Turgeon}$ à Québec auprès du gouverneur Thompson. $\mathrm{M}^{\mathrm{gr}}$ Bourget regrettait que les laïcs ne fissent rien. La première assemblée anti-unioniste eut finalement lieu à Québec le 17 janvier 1840 sous la présidence du journaliste John Neilson. Une requête circula, appuyée par les curés, ce qui choqua Thompson. À Montréal, la première assemblée eut lieu le 21 février 1840. Le projet de requête qui en résulta resta en plan, mais celle suscitée par $\mathrm{M}^{\mathrm{gr}}$ Lartigue et son clergé circula. Vital Têtu porta à Londres 100,000 signatures au bas des requêtes de Québec et de Montréal. $\mathbf{M}^{\mathrm{gr}}$ Bourget affirma à Thompson que le clergé appuyait la population contre le projet d'union, parce que ce projet était injuste, même si dans l'ensemble ce même clergé s'était opposé aux 
troubles de 1837-1838 qui avaient d'ailleurs mobilisé seulement une infime portion de la population canadienne.

\section{LA PLACE DE L'ÉGLISE ET DE LA RELIGION DANS LES COMPOSANTES DU PROCESSUS RÉVOLUTIONNAIRE DE $1837-1838$}

Au lendemain de l'échec de 1837-1838, le sentiment prevalait parmi les dirigeants de la société (en particulier parmi le clergé) d'avoir échappé à un immense danger ${ }^{24}$.

De quel danger s'agissait-il? C'était un processus révolutionnaire, c'est-à-dire un effort concerté de changement radical des structures sociopolitiques. La révolution patriote était soutenue et justifiée par une idéologie, un vaste système d'opinions reçues dans lesquelles les classes dominées du Bas-Canada se reconnaissaient entre elles et qui leur servaient de levier pour imposer dorénavant leur propre domination. Ainsi, les patriotes se ralliaient autour d'idées libérales et démocratiques qui leur assuraient le renversement de l'oppression anglaise.

Depuis 1830 , la crise économique créa moins de richesses. Donc, plus de gens de toutes extractions cherchaient à se les approprier. Les prix et les salaires diminuaient. Les profits aussi. Les commerçants s'en plaignirent, mais c'est la population qui écopa. Les boucs émissaires de tous ces malheurs furent vite désignés: les marchands qui manoeuvraient pour sauvegarder leurs profits, les immigrants qui menaçaient l'équilibre linguistique et augmentaient les concurrents dans la chasse aux emplois, et les instances gouvernementales qui favorisaient la grande bourgeoisie. Or, ces trois couches sociales, bien différentes à la vérité, avaient en commun d'être d'ascendance anglo-saxonne.

Les insurrections de 1837-1838 furent le résultat d'un mouvement nationaliste dominé par la petite bourgeoisie canadienne qui cherchait à défendre ses intérêts et à dominer la société, en utilisant un discours libéral et démocratique, voire républicain. En éliminant les étrangers venus après la conquête, les membres des professions libérales contribuaient non seulement à maintenir leur place au soleil, mais encore à prendre le haut du pavé, et même à définir les valeurs de leur nation libérée. Louis-Joseph Papineau était leur idole et leur porte-étendard.

\footnotetext{
29ernand OUELLET, «Les classes dominantes au Québec. 1760-1840. Bilan historiographique». RHAF. 38 n" 2 (automne 1984), p. 234.
} 
À l'intérieur de ce mouvement patriote, une aile gauche minoritaire, surtout regroupée à Mont iéal, visait plus que l'indépendance. Plusieurs leaders radicaux voyaient dans leur lutte politique une condition nécessaire à une révolution sociale plus profonde: des élections au suffrage universel, la citoyenneté à part entière pour les Amérindiens, l'abolition du régime seigneurial, l'éducation publique pour tous, la nationalisation des grands domaines terriens.

Fernand Ouellet estime que cette contradiction interne fut déterminante dans les événements de 1837-1838. "Dans ces circonstances, écrit-il, c'est le développement d'un conflit entre ces deux groupes à propos des objectifs sociaux de leur mouvement, qui aurait joué le plus fortement sur l'issue de la lutte armée»"

Mais plusieurs causes secondaires se conjuguèrent à celle-là pour acheminer la rébellion à un échec lamentable. L'organisation des insurgés fut déficiente à tous points de vue: aucune discipline parmi eux, pas suffisamment d'armes pour combattre, un très mauvais encadrement supérieur. Les rivalités entre chefs patriotes et certaines lâchetés individuelles expliquent aussi la rapidité de la débandade. La régionalisation du conflit, qui ne réussit jamais à s'étendre à la capitale provinciale, devint à la longue un handicap. Pourtant, malgré ces défaillances dans le leadership patriote, la pierre d'achoppement de la lutte se trouva dans le manque de solidarité entre les masses rurales et son élite cultivée.

Quel espace donner à la religion dans ce portrait? Le catholicisme était partie prenante de l'enjeu parce qu'il constituait un aspect de l'identité nationale. Il servait de rempart à la nation, face à la menace anglophone et protestante.

Mais cette religion, aux yeux des libéraux patriotes, ne structurait plus l'existence. Elle s'identifiait plutôt à un conformisme sociologique au service d'intérêts nationaux.

Ce désir de protéger l'intégrité des institutions traditionnelles du Canada français apparaît aussi dans les attitudes du nationalisme à l'égard de la religion et de l'État. Même si l'incroyance fait des progrès étonnants parmi l'élite canadienne-française du temps, même si l'esprit laïque ose se manifester avec vigueur en certaines occasions, on assiste cependant

"1) Ibid., p. 237. 
dans les milieux nationalistes à un curieux processus d identification de la religion à la nation."

Fait significatif à cet égard, les patriotes appuyèrent fermement $\mathrm{M}^{\mathrm{r}}$ Lartigue dans son débat avec les Sulpiciens français de Montréal pour l'établissement d'un diocèse distinct de Québec en 1836.

Par ailleurs, il existe une tension entre le nationalisme patriote et la religion catholique. Préoccupée du salut de son peupie et soucieuse d' obienir justice de la part de l'État, l'Église n'en demeurait pas moins fidèlement loyaliste. L'évêque de Montréal ne rejetait pas d'un bloc l'idéologie nationaliste qui cherchait à sauvegarder les coutumes, la langue et les lois de son pays. Mais il cherchait à se démarquer de l'emprise du libéralisme agnostique, sinon athée, qui se teintait de démocratie importée d'Europe.

En rapport avec les intérêts du clergé et en étroite connivence avec l'ultramontanisme grandissant, une idéologie cléricale mieux articulée donna la primauté à l'autorité dans l'Église et la société, en réaction aux désirs de changements véhiculés par l'idéologie libérale et démocratique des patriotes. Des libres penseurs canadiens remettaient en cause l'autorité établie, tant au niveau politique que religieux. Assimilant ces révoltés politiques aux hérétiques protestants, les chefs de l'église catholique préférèrent soutenir l'autorité civile établie, car elle garantissait la stabilité de l'ordre moral et le respect du bien commun comme celui de l'autonomie ecclésiastique chèrement acquise après 1760. Au fond, Dieu s'exprimait habituellement - selon les ultramontains - par l'autorité légitime, hiérarchiquement constituée, à laquelle tous devaient respect et obéissance.

La liberté religieuse, la fin de l'éducation confessionnelle et la séparation de l'Église et de l'État réclamées par les patriotes étaient, dans ce contexte, le prélude inévitable de la négation du principe d'autorité, germe de toute révolution sanglante. Transposé sur le plan religieux, le régime démocratique aurait permis, aux yeux des ultramontains, à tout croyant d'interpréter à sa guise la Révélation chrétienne et de faire fi de l'autorité du magistère chargé de l'interpréter adéquatement. Ainsi, selon $\mathrm{M}^{\text {rr }}$ Lartigue, la soumission aux supérieurs hiérarchiques civils et religieux était une attitude morale constituant une "partie intégrante du dépôt sacré de la foi ${ }^{32}$. En conséquence, refuser de se soumettre à l'État en 1837-1838, c'était

\footnotetext{
"Fernand OUELLET, «Nationalisme canadien-français et laïcisme au XIX" siècle". JeanPaul BERNARD, dir., Les idéologies québécoises au 19" siècle. Montréal, Boréal Express. 1973. p. 50.

"MEM, 1: 27, 8 janvier 1838 .
} 
renier Dieu qui avait transmis son pouvoir au gouvernement légitime établi dans le Bas-Canada, c'était refuser l'enseignement de la Bible corroboré par le magistère ecclésial, c'était littéralement s'excommunier de l'Église et renoncer au christianisme.

Marqués par les bouleversements de la révolution française, les tenants du discours clérical se refusèrent aux changements profonds souhaités par les patriotes et les chargèrent même de tous les maux du pays. L'ultramontanisme venait sacraliser l'autorité plutôt que le changement. La justification théologique de la fidélité à l'autorité établie se révéla être le facteur de dernière instance, facteur déterminant aux yeux des croyants par le fait même qu'il concernait le salut éternel d'un chacun.

L'échec de l'aventure patriote décapita le courant libéral et renforça l'ascendant clérical sur le peuple. Les soulèvements de 1837-1838 consacrèrent la défaite de l'élite laïque face au clergé canadien dans la lutte d'influence qu'ils se livraient depuis 1791. On s'attaquerait désormais aux transformations sociales par le biais d'une stratégie plus englobante: le Canada français avait le devoir moral et la mission providentielle de révéler le vrai Dieu à toute l'Amérique du Nord ${ }^{33}$.

${ }_{33}^{3}$ Louis ROUSSEAU, La prédication à Montréal de 1800 à 1830. Approche religiologique, Montréal, Fides, 1976, p. 237. 